\title{
Seguimiento del proceso de aprendizaje a través de la huella en Moodle y comparativa de resultados
}

\section{Begoña Peña $^{a}$ y Sara Pascual ${ }^{b}$}

Universidad de Zaragoza - Departamento de Ingeniería Mecánica (España) ae-mail: bpp@unizar.es, bsaraps@unizar.es

\begin{abstract}
The use of Virtual Learning Environments is widespread in most universities and it is also applied to other educational levels, such as Primary and Secondary schools. In fact, environments such as Moodle or Google Classroom are being essential tools to continue academic activities in those countries that, due to the pandemic of Covid-19, have suspended all face-to-face classes. In these educational platforms, all the activities carried out by each student are registerd and stored in detail. This information can be extracted as raw data or as indicators developed after automated analysis if the platform has the appropriate tools. In this work, the information extriacted for the subject of Engineering Thermodynamics from the last 3 academic years in the Degree in Engineering of Industrial Technologies is presented and compared. The results have been used to detect bad practices and study patterns, in order to improve assessment activities in particular and learning outcomes in general.
\end{abstract}

Keywords: Learning Analytics, Virtual Learning Environments, Higher Education Innovation, Engineering Thermodynamics.

\section{Resumen}

El uso de plataformas educativas digitales está ampliamente extendido en la mayoría de las universidades y también se empieza a aplicar en otros niveles educativos, como Primaria y Secundaria. De hecho, entornos como Moodle o Google Classroom están siendo herramientas esenciales para continuar con la formación académica en aquellos países que, debido a la pandemia del Covid-19, han tenido que suspender las clases presenciales. En estos" entornos de enseñanza virtual quedan registradas con detalle todas las actividades desarrolladas por cada estudiante. Esta información se puede extraer en forma de datos brutos o de indicadores tras un análisis automático si la plataforma dispone de las herramientas adecuadas. En es.te trabajo se presenta y compara la información extraída para la asignatura de Termodinámica Técnica de los últimos 3 cursos académicos en el Grado en Ingeniería de Tecnolgías Industriales. Los resultados se han utilizado para detectar malas prácticas e identificar patrones de estudio, con el fin de mejorar las actividades de evaluación en particular y los resultados de aprendizaje en general.

Palabras clave: Analíticas del Aprendizaje, Entornos Virtuales de Aprendizaje, Docencia Universitaria, Innovación, Termodinámica Técnica..

\section{Introducción}

El éxito en los resultados de aprendizaje y en la adquisición de competencias no sólo depende de los materiales y actividades planificados por el profesor, sino que depende en gran medida de cómo los estudiantes utilizan todos estos recursos. Los entornos virtuales de aprendizaje (Virtual Learning 
Environments, VLE), como Moodle o Google Classroom, permiten realizar un seguimiento continuo de la actividad de cada alumno y también detectar desviaciones generalizadas con respecto a los resultados esperados. Este tipo de plataformas educativas registran con detalle todos los movimientos del estudiante: descarga de materiales, resolución de cuestionarios, participación en foros, visualización de videos, etc.

Toda esta información puede analizarse con diferentes objetivos (Van Barneveld, 2012; Chatti, 2012): (i) educational data mining para establecer patrones de conducta y de aprendizaje o predecir el rendimiento del alumno (Xing, 2015; Tempelaar, 2015), (ii) academic analytics a nivel institucional para mejorar la calidad de los planes de estudio y adaptarse a los cambios socio-culturales observados en el alumnado (Macfadyen, 2012; Ferguson, 2016), (iii) educational action research para monitorizar y evaluar el proceso de aprendizaje con el fin de mejorarlo y garantizar la calidad (Clow, 2013), (iv) recommender systems para informar al alumno sobre su propio aprendizaje y dar recomendaciones para mejorar, (Gasevic, 2015; Boyer, 2016), (v) personalized adaptative learning para desarrollar entornos de aprendizaje personalizado.

Todas estas líneas de investigación se incluyen bajo la denominación genérica de Analíticas del Aprendizaje (Learning Analytics). Concretamente, esta área de conocimiento tiene como objetivo "la medición, recopilación, análisis e informe de datos sobre los estudiantes y sus contextos, con el fin de comprender y optimizar el aprendizaje y los entornos en los que se produce" (Ferguson, 2012). Esta disciplina aplica técnicas de big data o data mining (Clow, 2013) para convertir los datos registros en las plataformas digitales en información útil para describir (¿qué ha ocurrido?), predecir (¿qué ocurrirá?), diagnosticar (¿por qué ha sucedido?) y desarrollar estrategias de mejora en el proceso de aprendizaje (¿qué hacer en el futuro?).

El presente trabajo recoge y compara los resultados de analizar los registros de Moodle de los últimos 3 cursos académicos para la asignatura de Termodinámica Técnica y Fundamentos de Transmisión de Calor del Grado de Ingeniería en Tecnologías Industriales (GITI) de la Universidad de Zaragoza. Un número creciente de clases magistrales y la totalidad de las clases de laboratorio se desarrollan bajo el modelo de aula inversa, de manera que el alumno dispone de videos, textos y cuestionarios de autoevaluación para preparar la materia. En clase se aclaran dudas y se plantean y resuelven problemas y cuestiones en pequeños grupos. Para el resto de sesiones, aunque se sigue un modelo de clase magistral participativa, también se distribuye material complementario a través de Moodle. Además, como parte de la evaluación continua, se sincronizan tareas de aprendizaje y evaluación en forma de formularios, tareas y cuestionarios a través de la plataforma digital, de manera que se dispone de una gran cantidad de información digital de la actividad del estudiante (Peña, 2019). Para realizar el presente estudio, se han descargado los datos brutos en hojas de cálculo y se han analizado externamente, ya que la plataforma no disponde de herramientas específicas de LA.

\section{Objetivos}

Este trabajo ha tenido como objetivo general monitorizar el uso de los recursos proporcionados para el aprendizaje (vídeos, cuestionarios, textos), para comprender cómo estudian los alumnos, identificar malas prácticas y evaluar la eficacia de los procedimientos de evaluación como actividad formativa.

Para ello se han seguido los siguientes pasos:

- Recopilar datos sobre accesos, momentos de los accesos y resultados de aprendizaje para los cursos 2017-2018, 2018-2019 y 2019-2020. 
- Analizar los datos en base a los indicadores cuantitativos previamente identificados (Peña, 2019) sobre la dedicación y el esfuerzo promedio (¿qué ha ocurrido?).

- Comparar dichos indicadores para los últimos cinco cursos académicos para conocer los hábitos de estudo, cómo influyen en el éxito final y cómo pueden modificarse si fuese necesario (¿por qué ha ocurrido?).

- Establecer estrategias para mejorar el proceso de aprendizaje en el próximo curso (¿qué hacer en el futuro?).

\section{Desarrollo de la innovación}

\subsection{Contexto}

En este trabajo se ha analizado el proceso de aprendizaje y de evaluación en la asignatura de Termodinámica Técnica y Fundamentos de Transmisión de Calor, impartida en el tercer semestre $\left(2^{\circ}\right.$ curso) del Grado en Ingeniería de Tecnologías Industriales. Se trata de una asignatura troncal de 6 ECTS, equivalentes a 150 horas de trabajo, repartidas en 45 horas de clases presenciales de teoría y resolución de problemas, 15 horas de prácticas de laboratorio y 90 horas de trabajo personal del alumno.

Los recursos, metodologías y actividades de aprendizaje y evaluación se han ido modificando con el tiempo para tratar de conseguir una mayor implicación de los estudiantes en la asignatura y un aprendizaje más continuo y duradero. Los detalles sobre estos cambios pueden encontrarse en (Peña, 2019). El mayor cambio se realizó en el curso 2018-2019: la evaluación de trabajos tutorados pasó de realizarse con entrega de informes y resolución de cuestionarios en Moodle a calificarse mediante pruebas objetivas de tipo examen. Además se añadió un examen parcial a mitad de cuatrimestre que libera una parte de la materia en el examen final.

Por lo demás, la dinámica de las clases y los recursos se han mantenido en los tres cursos académicos: clases magistrales y de laboratorio que combinan aula inversa con clases convencionales participativas y resolución de problemas y trabajo autónomo guiado con actividades en Moodle.

El estudio aquí presentado ha tenido como objetivo comprobar que las medidas implementadas desde el curso 2018-2019 son efectivas para motivar el estudio continuo de la asignatura y' conseguir mejores resultados de aprendizaje.

\subsection{Recopilación de datos}

El entorno de aprendizaje virtual utilizado en la asignatura es Moodle, actualmente versión 3.5. No se dispone de ningún módulo específico sobre Analíticas del Aprendizaje, por lo que en trabajo se ha realizado a partir de los datos brutos descargados para cada curso y analizados en hojas de cálculo. Concretamente, se han calculado los siguientes indicadores:

- Actividad global por estudiante: evolución temporal de las vistas y mensajes obtenida del menú Administración, Informes, Estadísticas (Moodle, 2013)..

- Registros de acceso a los videos: porcentaje de alumnos, número de visualizaciones promedio, evolución temporal de los accesos.

- Registros de acceso a los cuestionarios de autoevaluación: porcentaje de participantes, número de intentos promedio y evolución temporal de los intentos.

El procedimiento para obtener los registros de cada actividad (videos y cuestionarios en este caso) se obtiene del menú Administración, Informes, Registros, seleccionando el recurso concreto (Peña, 2019).

(cc) EY-NC-ND 2020, Universitat Politècnica de València

Congreso In-Red (2020) 


\section{Resultados}

\subsection{Resultados de aprendizaje}

En la Tabla 1 se recogen y comparan los datos de cada curso analizado: número de estudiantes matriculados, calificaciones promedio en diferentes actividades y porcentaje de estudiantes aprobados en la primera convocatoria. La nota de cuestionarios y trabajos tutorizados que aparece desglosada para el curso 2017-2018, aparece agrupada para los dos cursos posteriores porque ambas actividades se han evaluado a través de las mismas pruebas presenciales.

La nota media de los estudiantes aprobados es idéntica durante los tres cursos analizados. El porcentaje de alumnos presentados ha ido disminuyendo progresivamente desde el $90 \%$ hasta el $84 \%$. Sin embargo, el porcentaje de éxito ha cambiado significativamente, aunque en todos los casos ha estado acorde a los registros de Moodle y a lo observado por la profesora durante el cuatrimestre.

Los peores resultados son para el curso 2017-2018. Se registró una baja participación en las tutorías y se detectaron múltiples copias en la realización de los trabajos tutorizados, con calificaciones muy elevadas en los mismos pero baja tasa de éxito en el examen final. Los mejores resultados finales se obtuvieron para el curso 2018-2019, con más de un 60\% de aprobados en la primera convocatoria. En este curso, se observó una dinámica positiva en clase, colaboración muy activa entre alumnos, asistencia a tutorías en grupo y seguimiento continuo por parte de un elevado número de estudiantes durante todo el semestre. Para el curso actual el porcentaje de aprobados se ha reducido prácticamente en 8 pp respecto al curso anterior, pese a ser un grupo mucho más reducido. La asistencia a tutorías ha sido muy escasa y se ha detectado un ambiente de baja colaboración entre los alumnos y baja motivación por aprender.

Tabla 1. Comparación de los resultados de aprendizaje

\begin{tabular}{lccc}
\hline \multicolumn{1}{c}{ Curso } & $\mathbf{2 0 1 7 - 2 0 1 8}$ & $\mathbf{2 0 1 8 - 2 0 1 9}$ & $\mathbf{2 0 1 9 - 2 0 2 0}$ \\
\hline Número de alumnos matriculados & 52 & 62 & 43 \\
Nota en cuestionarios de Moodle & 7,3 & & \\
Nota de trabajos tutorados & 7,7 & 7,1 & 6,8 \\
Calificación (1 ${ }^{\text {a }}$ Convocatoria) & 6,4 & 6,4 & 6,4 \\
Estudiantes presentados (\%) & 90,4 & 87,1 & 83,7 \\
Estudiantes aprobados (\%) & 45,8 & 61,1 & 52,8 \\
\hline
\end{tabular}

Con el fin de analizar las causas de esos resultados, se presentan a continuación diversos indicadores de seguimiento de la asignatura, obtenidos de los registros de Moodle.

\subsection{Actividad global en la plataforma}

En esta sección se analizan las Vistas y Mensajes que Moodle proporciona en el menú Administración, Informes, Estadísticas (Moodle, 2013). Las Vistas engloban actividades pasivas del usuario, como accesos o visualización de recursos o descargas. Los Mensajes incluyen acciones activas del usuario, como participación en foros, envío de tareas, resolución de cuestionarios o encuestas.

En las gráficas de la Figura 1 se muestran las Vistas y Mensajes por estudiante matriculado durante los tres últimos cursos a lo largo del semestre (septiembre-febrero). Hay que tener en cuenta que los datos propocionados por Moodle se obtienen agrupados por semanas. 
En consonacia con las percepciones de la profesora antes mencionadas, se observa un patrón de uso bastante diferente en cada curso. En el curso 2017-2018, se observa un uso muy bajo de los recursos durante todo el cuatrimestre tanto en Vistas como en Mensajes.

En los cursos 2018-2019 y 2019-2020 los accesos están condicionados principalmente por las fechas de las actividades de evaluación. Con respecto a las Vistas, en los dos últimos cursos se aprecian dos picos intermedios asociados a las pruebas de evaluación de los trabajos tutorizados y un pico más pronunciado al final del semestre motivado por los exámenes de enero (prueba de trabajos y examen final).
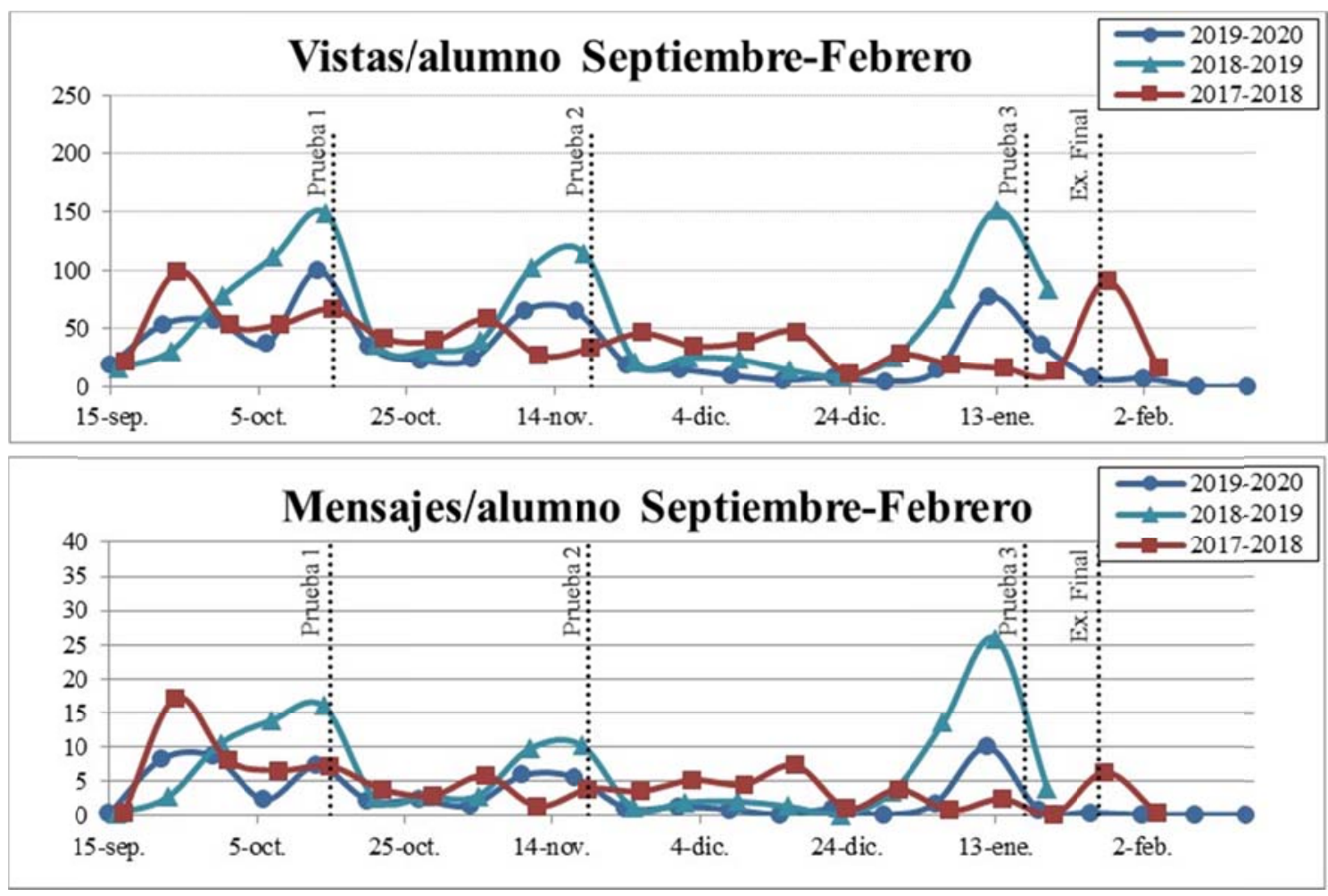

Fig. 1 Actividad general por alumno registrada en los cursos de Moodle.

En el curso 2018-2019, se observa también un patrón similar en los mensajes, lo que se debe principalmente a la realización de cuestionarios de autoevaluación. Sin embargo, los picos para el curso 2019-2020 apenas se aprecian, siendo mayor el que correponde al examen final.

\subsection{Visualización de videos docentes}

Para la impartición de ciertas clases magistrales bajo el modelo de aula inversa y para la preparación de prácticas, se han utilizado un total de 21 videos docentes en la asignatura (Zabalza, 2016-2017): 8 videos recomendados especialmente para las sesiones de prácticas (B1), 6 orientados a las clases del Tema 2, impartidas bajo aula inversa, (B2) y 7 videos más disponibles en el canal de YouTube de la Universidad Politécnica de Valencia que se recomiendan para los últimos temas del curso (B3). 
Tabla 2. Resumen del uso de los videos docentes

\begin{tabular}{llccc}
\hline Bloque & Indicador & $\mathbf{2 0 1 7 - 2 0 1 8}$ & $\mathbf{2 0 1 8 - 2 0 1 9}$ & $\mathbf{2 0 1 9 - 2 0 2 0}$ \\
\hline B1- prácticas & E (\%) & 59.6 & 83.5 & 77.0 \\
& A/E & 1.1 & 1.6 & 1.4 \\
B2- Tema 2 & E (\%) & 90.7 & 92.2 & 89.5 \\
& A/E & 3.1 & 2.7 & 4.4 \\
B3 - Externos & E (\%) & 33.2 & 31.1 & 18.3 \\
& A/E & 0.5 & 0.5 & 0.3 \\
\hline
\end{tabular}

El porcentaje de alumnos que ha accedido al menos una vez, E (\%), y el número de accesos promedio por estudiante que ha accedido al menos una vez a cada video (A/E) se comparan en Tabla 2 para los tres cursos analizados. Globalmente, se observa un mayor porcentaje de estudiantes en el curso 2018-2019, aunque en el bloque B2 el número de consultas por estudiante es superior para el curso actual. Según se ha visto, ha habido unos pocos estudiantes que han realizado un número extrañamente alto, que han podido falsear los resultados medios.

Los videos del bloque B2 son los que mejor acogida tienen entre los alumnos en todos los cursos aquí estudiados y también en cursos previos (Peña, 2019). Esto se debe a que el tema al que corresponden se imparte bajo el modelo de aula inversa, de manera que para poder realizar las actividades planificadas en el aula es imprescindible haber preparado la materia con textos y con esos videos. Con respecto al bloque B1, se observa un aumento significativo en los dos últimos cursos, motivado por la realización obligatoria desde el curso 2018-2019 de un cuestionario al comienzo de cada práctica que determina si el alumno puede o no realizarla ese día en función de su preparación previa. Los videos del bloque B3, que se recomiendan intensamente para los temas finales continúan, sin tener la acogida esperada, reduciéndose en 15 puntos porcentuales la fracción de estudiantes con al menos un acceso en el curso actual.

Para conocer los hábitos de estudio de los estudiantes, se ha analizado la evolución temporal de los registros. En la Figura 2 se han representado, agrupados por bloques, los accesos totales a cada video para el curso actual. El patrón observado es similar al obtenido para el curso 2018-2019 (Peña, 2019), aunque con una participación más baja.

A pesar de que los videos del bloque B1 han sido recomendados también para la preparación de las pruebas de evaluación, se observa que han sido utilizados esencialmente para la preparación de las prácticas. La curva de cada video presenta claramente dos picos que coinciden con las fechas de realización de las prácticas, a excepción del video 3 que solo presenta un pico de visualización correpondiente a la primera sesión de la segunda práctica.

Los videos del bloque B2 se han utilizado para la preparación de las clases presenciales del Tema 2, impartidas entre el 24 de septiembre y el 7 de octubre, y para la prueba parcial del 15 de octubre. El video 14 muestra un repunte de visualizaciones entre el 22 y 27 de octubre debido a su recomendación para la segunda práctica realizada el 25 y el 29 de octubre.

La visualización de los videos del bloque B3 ha sido muy baja y ha estado asociada a la impartición en diciembre de la materia relacionada y a la proximidad del examen final. Igual que en cursos anteriores, el hecho de recomendar vídeos de otras universidades parece desmotivar su uso por considerarse complementario. 
De la Figura 2 se puede concluir que el uso de los videos está muy determinado por la existencia o no de pruebas de evaluación inminentes y además directamente relacionadas con dichos videos. Este patrón se observó también en el curso 2018-2019 para los tres bloques (Peña, 2019). Este comportamiento era esperable, pero llama la atención que sea tan acusado y que no vuelvan a utilizarse para otras pruebas de evaluación, como el examen final.

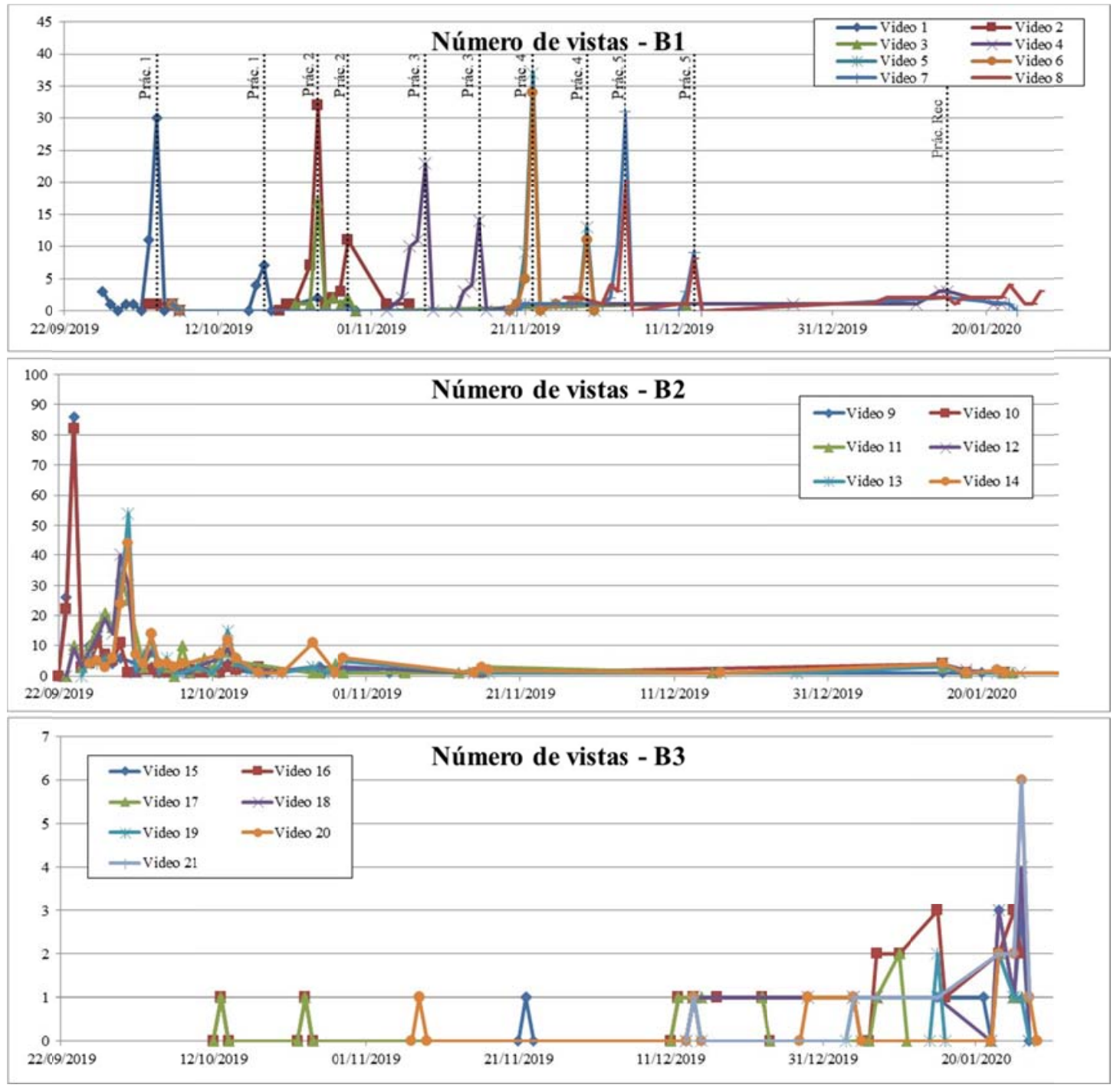

Fig. 2 Uso de los videos a lo largo del cuatrimestre del curso 2019-2020.

\subsection{Uso de los cuestionarios de autoevaluación}

Al finalizar cada tema, se habilita un cuestionario de autoevaluación constituido por preguntas que incluyen retroalimentación sobre los conceptos y procedimientos estudiados. En el curso 2017-2018, la calificación promedio de dichos cuestionarios constituía un 5\% de la nota final y el alumno disponía de 3 intentos contando únicamente la nota del último. Posteriormente se podían utilizar libremente como herramienta de aprendizaje y autoevaluación sin límite de intentos (Peña, 2018). Sin embargo, se observó una calificación muy alta y una baja tasa de éxito en las pruebas de evaluación, indicando copia fraudulenta. 
Por esta razón, en los dos últimos cursos se han dejado sólo como herramienta de aprendizaje y autoevaluación. Para motivar su realización, parte de esas preguntas se incluyen (literalmente) en las tres pruebas objetivas realizadas durante la evaluación continua.

En la Tabla 3 se compara el porcentaje de estudiantes que realizaron al menos un intento (E) y el promedio de intentos por estudiante y cuestionario (I/E).

Tabla 3. Resumen sobre los cuestionarios de autoevaluación

\begin{tabular}{ccc}
\hline Curso & E (\%) & I/E \\
\hline $2017-2018$ & 25,2 & 1,3 \\
$2018-2019$ & 75,8 & 3,1 \\
$2019-2020$ & 71,5 & 2,1 \\
\hline
\end{tabular}

En el curso 2018-2019 se observa la mayor participación y mayor número de intentos por estudiante. Tres cuartas partes de los estudiantes realizaron un promedio de 3 intentos por cuestionario, lo que supuso un aumento muy significativo con respecto al curso previo, en el que tan solo el $25 \%$ realizó intentos. Respecto al curso actual, el porcentaje de estudiantes que han realizado al menos un intento se ha mantienido en cifras similares a las del curso 2018-2019. Sin embargo, el número de intentos por estudiante se ha reducido en un $30 \%$.

La Figura 3 muestra el uso de los cuestionarios a lo largo del semestre. Igual que sucede con los videos, los cuestionarios han sido utilizados en días previos a la realización de las pruebas de evaluación de los trabajos tutorizados en los que las preguntas se extraen de los mismos. Sin embargo, no se aprecia apenas actividad en días previos al examen final. Este mismo comportamiento se observó también en el curso 2018-2019 en el que el procedimiento de evaluación fue idéntico, aunque la participación fue más alta como ya se observa en la Tabla 3.



Fig. 3 Uso de los cuestionarios de autoevaluación a lo largo del cuatrimestre. 


\section{Conclusiones}

En este trabajo se han analizado y comparado los registros de los estudiantes en Moodle y los resultados de aprendizaje para los tres últimos cursos académicos en la asignatura de Termodinámica Técnica y Fundamentos de Transmisión de Calor del grado en Ingeniería de Tecnologías Industriales. Concretamente, se ha analizado el uso de vídeos y de cuestionarios de autoevaluación en base a magnitudes estadísticas y en función del tiempo, ya que se habían identíficado como magnitudes relevantes para el éxito en el examen final. Las diferencias encontradas se han interpretado en base a los resultados en las actividades de evaluación y a la actitud observada en los estudiantes.

Las conclusiones derivadas del trabajo se recogen a continuación:

- El análisis durante varios cursos académicos de los registros de utilización de recursos como vídeos o cuestionarios puede servir para comprobar el uso y la utilidad de los mismos en el proceso de aprendizaje.

- Una vez comprobada su eficacia, la monitorización continua de la actividad de los estudiantes a través de ciertos indicadores, como los analizados en este trabajo (actividad general, las visualizaciones de vídeos y los intentos en los cuestionarios de autoevaluación), resulta útil para que el profesor compruebe la buena marcha del aprendizaje, al menos en promedio, y pueda realizar avisos a tiempo si se observa desmotivación entre los estudiantes.

- A nivel individual, esos indicadores podrían utilizarse para detectar abandonos o usos inapropiados y realizar una intervención para revertir la situación. Sin embargo, esto puede suponer una carga de trabajo importante para el profesor si el Entorno Virtual de Aprendizaje no proporciona informes simplificados.

- Hay que señalar, que además de una buena planificación de las actividades y de la selección adecuada de recusos, resulta esencial la correcta actuación de los estudiantes, que en muchos casos queda fuera del alcance del profesor por estar condicionada por agentes externos (sobrecarga de trabajo en otras asignaturas, dinámicas negativas en la clase, etc.).

En definitiva, el análisis de los registros de la actividad en Moodle es una herramienta potente para detectar malos hábitos de estudio, realizar acciones correctoras cuando se detectan desviaciones y adaptar los sistemas de evaluación para fomentar el seguimiento continuo de la asignatura y el buen uso de los recursos de aprendizaje.

\section{Agradecimientos}

Las autoras agradecen a los profesores M Belén Zalba Nonay e Ignacio Zabalza Bribián su disposición para discutir y compartir experiencia en materia de innovación docente. Este trabajo se ha desarrollado dentro del proyecto de innovación docente PIIDUZ_19_265 (Programa de Proyectos de Innovación Docente para Grupos de Profesores del Vicerrectorado de Política Académica de la Universidad de Zaragoza).

\section{Referencias}

BOYER, A. y BONNIN, G. (2016). Higher Education and the Revolution of Learning Analytics. Report of the International Council for Open and Distance Education (ICDE). En: <https:/icde. memberclicks.net/assets/RESOURCES/anne_la_report\%20cc\%20licence.pdf $>$ [Consulta: 9 de febrero de 2019]. 
CHATTI, M.A., DYCKHOFF, A.L. SCHROEDER, U. y THÜS, H. (2012). A reference model for learning analytics. Int. J. Technology Enhanced Learning. 4 (5-6) pp. 318-331. Disponible en: $<$ https://www.thues.com/upload/pdf/2012/CDST12_IJTEL.pdf $>$ [Consulta : 29 de mayo de 2019].

CLOW, D. (2013). An overview of learning analytics. Teaching in Higher Education, 18 (6) pp. 683-695. Disponible en: <http://oro.open.ac.uk/38237/> [Consulta : 29 de mayo de 2019]. doi: 10.1080/13562517.2013.827653.

FERGUSON, R. (2012). Learning analytics: drivers, developments and challenges. Int. J. of Technology Enhanced Learning, $\quad 4 \quad(5-6) \quad$ pp. $\quad 304-317 . \quad$ Disponible en: <http://oro.open.ac.uk/36374/1/IJTEL40501_Ferguson\%20Jan\%202013.pdf> [Consulta : 29 de mayo de 2019]. doi:10.1504/IJTEL.2012.051816.

FERGUSON, R., et al. (2016). Research Evidence on the Use of Learning Analytics - Implications for Education Policy. R. Vuorikari, J. Castaño Muñoz (Eds.). Joint Research Centre Science for Policy Report; EUR 28294 EN; doi:10.2791/955210.

GASEVIC, D., DAWSON, S. y SIEMENS, G. (2015). Let's not forget: Learning analytics are about learning. TechTrends, 59 (1) pp. 64. doi: 10.1007/s11528-014-0822-x.

MACFADYEN, L.P. y DAWSON, S. (2012). Numbers Are Not Enough. Why e-Learning Anallytics Failed to Inform an Institutional Strategic Plan. Educational Technology \& Society 15(3) pp149-163.

MOODLE (2013). Course overview report. Documentación de Moodle.org. $<$ https://docs.moodle.org/25/en/Course_overview_report > [Consulta: 9 de febrero de 2019].

PEÑA, B. et al. (2018). "Experiencia piloto de aula invertida para mejorar el proceso de enseñanza-aprendizaje en la asignatura de Termodinámica Técnica”. En: Actas del congreso INRED 2018, Valencia, Editorial Universitat Politècnica de València. Disponible en: <http://ocs.editorial.upv.es/ index.php/INRED/INRED2018/paper/viewFile/8583/4153> [Consulta: 9 de febrero de 2019]. doi: 10.4995/INRED2018.2018.8583.

PEÑA, B. (2019). “Análisis del aprendizaje a través de la huella en Moodle: aplicación en la asignatura de Termodinámica Técnica”. En: Actas del congreso INRED 2019, Valencia, Editorial Universitat Politècnica de València. Disponible en: <http://ocs.editorial.upv.es/index.php/INRED/INRED2019/paper/view/10415/4750> [Consulta: 21 de marzo de 2020]. doi: 10.4995/INRED2019.2019.10415.

SLADE, S. y PRINSLOO, P. (2013). Learning Analytics: Ethical Issues and Dilemmas. American Behavioral Scientist 57 (10) pp. 1510-1529. doi: 10.1177/0002764213479366.

TEMPELAAR, D.T., RIENTIES, B.C. y GIESBERS, B. (2015). In search for the most informative data for feedback generation: Learning analytics in a data-rich context, Computers in Human Behavior 47 pp. 157-167. doi: 10.1016/j.chb.2014.05.038.

VAN BARNEVELD, A., ARNOLD, K. E., y CAMPBELL, J. P. (2012). Analytics in Higher Education: Establishing a Common Language. ELI White Papers, (1/2012). Disponible en: <https://library.educause.edu//media/files/library/2012/1/eli3026-pdf.pdf> [Consulta : 29 de mayo de 2019].

XING, W., GUO, R., PETAKOVIC, E. y GOGGINS. S. (2015). Participation-based student final performance prediction model through interpretable Genetic Programming: Integrating learning analytics, educational data mining and theory. Computers in Human Behavior 47 pp. 168-181.

ZABALZA, I., PEÑA, B., LLERA, E.M. y USÓN, S. (2016) "Improving the teaching-learning process using educational videos as reusable learning objects in the field of thermal engineering", Proceedings of the 8th International Conference on Education and New Learning Technologies (EDULEARN 2016), Barcelona, IATED Academy, pp. 363-372. doi: 10.21125/edulearn.2016.1068.

ZABALZA, I., et al. (2017) "Development of educational videos as reusable learning objects for their integration into an Open Courseware on fundamentals of thermodynamics and thermal engineering", En: INTED17 Proceedings of the 11th annual International Technology, Education and Development Conference, Valencia, IATED Academy, pp. 4453-4461. doi: 10.21125/inted.2017.1055. 